\title{
Philonsorbonne
}

9 | 2015

Année 2014-2015

\section{Acte et motivation dans la philosophie morale de Spinoza}

\section{Steven NADLER}

Traducteur : Sophie Laveran

\section{OpenEdition \\ Journals}

Édition électronique

URL : https://journals.openedition.org/philonsorbonne/751

DOI : 10.4000/philonsorbonne.751

ISSN : 2270-7336

Éditeur

Publications de la Sorbonne

\section{Édition imprimée}

Date de publication : 1 janvier 2015

Pagination : 149-168

ISSN : 1255-183X

\section{Référence électronique}

Steven NADLER, « Acte et motivation dans la philosophie morale de Spinoza », Philonsorbonne [En ligne], 9 | 2015, mis en ligne le 11 janvier 2015, consulté le 08 juin 2021. URL : http://

journals.openedition.org/philonsorbonne/751; DOI : https://doi.org/10.4000/philonsorbonne.751

(c) Tous droits réservés 


\title{
Acte et motivation dans la philosophie morale de Spinoza
}

\author{
Steven NADLER
}

Dans son œuvre maîtresse, l'Éthique, Spinoza a beaucoup à dire sur la vertu, le bien, le désir, l'action et le bonheur - en un mot, sur tous les grands thèmes de la philosophie morale. Il soutient également la thèse du caractère résolument égoïste de la motivation. Toute l'activité humaine (et même, toute activité d'un individu de la nature, quel qu'il soit) dérive de l'effort de chacun pour persévérer dans son être et maximiser sa puissance d'agir. Cependant, même si Spinoza rejette l'évaluation morale de l'action en termes de «bien» ou de «mal », il croit en l'existence de critères normatifs permettant de juger les actions, selon qu'elles contribuent de fait à l'effort égoïste de l'agent, et à quel degré. Apparemment, il faudrait en conclure que les motivations de l'agent ne jouent aucun rôle dans la qualification morale de l'action (bien qu'elles soient importantes pour la qualification de l'agent lui-même), principalement parce que tous les agents, sans exception, ont la même et unique motivation fondamentale - leur intérêt propre - et qu'une action a pour effet, soit de contribuer, soit de ne pas contribuer à l'accroissement de la puissance de l'agent, et ce, indépendamment de sa motivation.

Toutefois, comme c'est souvent le cas chez Spinoza, les choses sont beaucoup plus compliquées qu'il n'y paraît. En réalité, Spinoza admet qu'une même action mérite, au-delà de sa « performance », de ses effets, une évaluation morale différente, fondée sur la motivation, qui équivaut, pour lui, aux déterminations causales de l'esprit. Dans cet article, après une présentation générale de quelques éléments essentiels de la philosophie morale de Spinoza, j'examinerai comment, malgré l'égoïsme universel qui la fonde, son éthique laisse une place à la discrimination normative des 
motivations, ainsi qu'à la prise en compte de ces motivations dans l'évaluation morale des actions qui en découlent.

\section{L'égoïsme}

Dans la troisième partie de l'Éthique, après avoir expliqué les fondements métaphysiques et épistémologiques de la nature humaine, Spinoza s'intéresse de près à ce qu'il appelle alternativement « la puissance d'agir (potentia agendi) » ou «la force d'exister (vis existendi) ». Chaque chose singulière, dans la nature, c'est-à-dire chaque mode fini de la substance éternelle, nécessaire et infinie, est une expression partielle et limitée de la même puissance infinie, celle de Dieu ou de la Nature. Cette puissance infinie de la Nature se manifeste comme esprits individuels dans l'attribut Pensée et comme corps individuels dans l'attribut Étendue (qui sont les deux seuls attributs divins que nous connaissons). Chaque esprit individuel est une expression finie de la puissance infinie de penser de Dieu, ou de la Nature; de même, chaque corps particulier est une expression de la puissance infinie de Dieu ou de la Nature en tant que matière et mouvement. Cette quantité finie de puissance qui constitue chaque chose singulière est ce que Spinoza appelle conatus, que l'on peut traduire par « effort » ou «tendance ».

Pour toute chose particulière, cette détermination finie de puissance se manifeste comme un effort pour persévérer dans cette individualité : être.

Chaque chose, autant qu'il est en elle, s'efforce de persévérer dans son

DEMONSTRATION. En effet, les choses singulières sont des manières, par lesquelles s'expriment les attributs de Dieu de manière précise et déterminée (par le Coroll. Prop. 25 p. 1), c'est-à-dire (par la Prop. 34 p. 1) des choses qui expriment de manière précise et déterminée la puissance de Dieu, par laquelle Dieu est et agit ; et nulle chose n'a en soi rien qui puisse la détruire, autrement dit, qui supprime son existence (par la Prop. 4 de cette p.) ; mais, au contraire, elle s'oppose à tout ce qui peut supprimer son existence (par la Prop. précéd.), et, par suite, autant qu'elle peut, et qu'il est en elle, elle s'efforce de persévérer dans son être ${ }^{1}$.

Il y a, dans toute chose - corps ou esprit -, une sorte d'inertie d'existence par laquelle elle résiste à toute tentative de la détruire ou de la changer en pire. Ce n'est pas une caractéristique temporaire ou accidentelle de la chose, sans laquelle cette chose pourrait être ; ce n'est pas non plus quelque chose qui ne se manifeste qu'en la présence d'une force contraire. C'est plutôt un effort positif et constant de l'individu pour maintenir et

1. Spinoza, Éthique, III, 6, trad. fr. B. Pautrat, Paris, Seuil, 1988. 
même augmenter sa puissance, qui enveloppe «une durée indéfinie ${ }^{2}$ » et opère au cœur de l'individuation de la chose.

Spinoza définit les affects, d'une manière générale, comme des changements du conatus, comme des transitions ou passages à une plus grande ou au contraire à une moindre puissance de l'effort. Les affects passifs, ou passions, sont les transitions de la puissance d'un individu qui sont causées par des choses extérieures : la joie (laetitia), par exemple, est le passage à une plus grande puissance d'agir, tandis que la tristesse (tristitia) est le passage à une moindre puissance d'agir provoqué par un objet. De tels changements de puissance constituent le fondement des motivations des agents et de ce qu'ils font. Le fait qu'ils recherchent certaines choses, qu'ils en évitent d'autres, leurs décisions, leurs jugements sur le bien et le mal, tout cela est motivé par la joie et la tristesse, l'amour et la haine, le plaisir et la douleur, autrement dit par les modifications de l'effort pour persévérer dans l'être. Nous recherchons certaines choses parce que nous les aimons, et nous les aimons parce que nous sommes conscients qu'elles provoquent un accroissement de nos capacités. De la même manière, nous évitons certaines choses parce que nous les haïssons, et nous les haïssons parce que nous sommes conscients qu'elles provoquent une diminution de nos capacités :

Tout ce que nous imaginons contribuer à la Joie, nous nous efforçons de le promouvoir pour que cela se fasse ; et ce que nous imaginons y être contraire, autrement dit contribuer à la Tristesse, nous nous efforçons de l'éloigner ou de le détruire ${ }^{3}$.

Plus précisément, dans l'esprit humain, le conatus d'un individu se manifeste comme «volonté »: non pas une faculté de vouloir abstraite, mais l'ensemble des affirmations ou négations mentales particulières qui constitue l'essentiel de la vie mentale. Lorsque l'être humain est considéré comme une entité composite, constituée d'un esprit et d'un corps, son conatus consiste dans l'«appétit». Lorsqu'une personne est consciente de l'effort conjoint de son esprit et de son corps, c'est-à-dire lorsqu'elle a conscience de son appétit, il devient «désir ${ }^{4} »$. Dans le cas de l'esprit comme dans celui du composé de corps et d'esprit, le conatus en tant que désir est la force de motivation qui est à la racine des efforts de la personne ${ }^{5}$. Notre désir et notre

2. Ibid., III, 8 .

3. Ibid., III, 28. Tous les individus, dans la nature, pâtissent d'affects ou de changements dans leur conatus; cependant, Spinoza, dans son analyse des passions et de leur rôle dans la motivation, se limite principalement aux manifestations du conatus chez les êtres humains. Et dans son étude de l'être humain, son attention se porte principalement sur les phénomènes mentaux, même si chaque expression mentale aura nécessairement son corrélat corporel, en vertu du parallélisme esprit-corps.

4. Ibid., III, 9, scolie.

5. On peut supposer que, dans le corps humain, le conatus se manifeste par la capacité du corps à résister à tout ce qui tend à changer le rapport de mouvement et de repos entre ses parties, dont l'horizon est sa dissolution. 
effort se portent toujours nécessairement vers les choses que nous croyons favorables à notre bien-être et au bien-être du corps dont dépend notre existence. La motivation, dans un sens large, consiste dans une combinaison de croyance et de désir. Mon désir pour $x$, qui me pousse à rechercher $x$, est déterminé, guidé par ma croyance que $x$ m'apportera de la joie, et augmentera ma puissance - et notons que pour Spinoza, le désir, en tant qu'expression du conatus, est la seule force de motivation. Si je tends à faire $x$, c'est parce que je désire nécessairement faire ce qui m'est le plus profitable, et parce que je crois que $x$ est ce qui m'est le plus profitable ${ }^{6}$.

Selon Spinoza, les êtres humains sont donc des agents profondément égoïstes. Une personne n'est poussée à rechercher ou éviter ceci ou cela qu'en vertu des effets positifs ou négatifs de la chose tels qu'ils sont perçus, autrement dit, en vertu des croyances de la personne concernant la manière dont cette chose contribue à son projet d'autoconservation. L'effort de persévérance est prévalent. Il constitue «l'essence même de l'homme, de la nature de qui suivent nécessairement les actes qui servent à sa conservation $^{7} ~$. Ainsi, écrit-il, « l'homme est déterminé à les faire ${ }^{8}$ [les actes qui servent à sa conservation] ». Cet égoïsme est similaire à celui de Hobbes, qui l'a vraisemblablement influencé (Spinoza a lu ses travaux dans les années 1660, lors de la rédaction de l'Éthique), et qui affirmait dans le Léviathan que «l'objet des actes volontaires de chacun est un bien pour soi-même ${ }^{9} »$.

Jusqu'à présent, néanmoins, Spinoza ne nous présente qu'un égoïsme psychologique : chaque agent fait toujours nécessairement ce qu'il croit être dans son intérêt, ce qu'il estime être le mieux à même de lui apporter de la

6. J'attribue donc à Spinoza un modèle de motivation «croyance/désir »; pour une discussion générale de ce modèle, voir S. Sverdlik, Motive and Rightness, chap. 2, Oxford, Oxford University Press, 2011. En distinguant ici entre croyance et désir, je déforme délibérément la conception de Spinoza, pour simplifier l'explication (et éviter de présenter sa psychologie des idées). À proprement parler, la croyance (qu'elle soit une idée de la raison ou une idée dérivée de l'expérience sensible, ou imagination) est, en tant qu'événement mental, identique au désir. Garrett établit bien ce point : « Si, par exemple, un individu détermine par la raison que son propre avantage réside dans la recherche de la connaissance... alors l'idée qui constitue cette compréhension sera elle-même un désir pour la chose ainsi conçue, selon Spinoza. Elle ne sera pas simplement ce qui dirige ou stimule un tel désir ; elle sera ce désir (If, for example, one determines by reason that one's own advantage lies in the pursuit of knowledge... then the idea that constitutes this understanding will itself be a desire for the thing so conceived, in Spinoza's view. It will not merely direct or stimulate such a desire; it will be such a desire)». («Spinoza's Ethical Theory», in The Cambridge Companion to Spinoza, D. Garrett (dir.), Cambridge, Cambridge University Press, 1996, p. 296). Pour Spinoza, la raison peut motiver l'action, dans l'exacte mesure où croire que $x$ est bon (avoir une idée qui représente $x$ comme conduisant à son propre bien-être) est la même chose que désirer $x$.

7. Éthique, op. cit., III, 9, scolie.

8. Ibid.

9. Léviathan, XIV, 8. Pour une comparaison entre Spinoza et Hobbes sur cette question, voir E. M. Curley, Behind the Geometric Method, chap. 3, Princeton, Princeton University Press, 1988. Pour la discussion sur les passions en général chez Hobbes (qui est remarquablement similaire à l'analyse de Spinoza à d'importants égards), voir Léviathan, VI. 
joie et d'accroître sa puissance d'agir (et corrélativement, chaque agent évite toujours nécessairement ce qu'il croit aller à l'encontre de son intérêt, c'est-à-dire ce qui, selon lui, va diminuer sa puissance d'agir $\left.{ }^{10}\right)$. Les choses deviennent plus intéressantes, d'un point de vue éthique, lorsque nous nous intéressons à la définition que donne Spinoza des termes moraux tels que le «bien » ou la « vertu».

\section{Le bien}

Spinoza commence la quatrième partie de l'Éthique par une préface dans laquelle il étudie le langage éthique de base : bien et mal, parfait et imparfait. Il insiste sur le fait que ces termes ne désignent pas des caractéristiques absolues et objectives des choses, des propriétés qu'elles détiendraient indépendamment d'autres choses, et en particulier des agents humains. Ce qui suit du naturalisme de Spinoza, c'est qu' aucune chose, prise en elle-même et sans relation avec autre chose, n'est bonne ou mauvaise, parfaite ou imparfaite, et encore moins lorsque ces mots sont compris dans leur sens normatif. Ce qui est, est, point final. Pour le dire autrement, tout ce qui existe, si on le considère en soi, est parfait à quelque degré ; la perfection est un terme ontologique qui doit simplement être compris comme un synonyme de réalité, ou encore de puissance de persévérance dans l'être. Lorsque nous disons qu'une chose a une perfection dans ce sens descriptif, cela n'implique aucune évaluation: " toutes [les choses], en cela, sont égales », écrit Spinoza à la fin de cette préface, ce qui n'implique pas que toutes les choses ont le même degré de réalité ou de puissance, mais plutôt que toute chose est, simplement, un degré positif de réalité ou de puissance.

Que désignent donc les termes «bien», «mal» (et «parfait», « imparfait»), dans leur sens normatif, s'il ne s'agit pas de caractéristiques objectives, indépendantes de l'esprit humain? Ils désignent une mesure qui

10. Il semble que, pour Spinoza, une bonne description du contenu des croyances qui me motivent ne devrait pas être «faire cette action ou rechercher cette chose m'apportera un accroissement de puissance ou contribuera à ma préservation », en particulier parce que, d'un point de vue empirique, cela semble hautement improbable. Spinoza semble plutôt suggérer que je suis poussé à faire les choses que j'associe à la joie et à éviter celles que j'associe à la tristesse ; ceci est beaucoup plus plausible que soutenir que je suis mû par le désir de persévérer dans l'être. Je suis poussé à être généreux envers mes enfants et à contribuer au bien-être de mes amis parce que, dans mon esprit, ces choses sont associées à la joie. Et puisque la joie est justement l'accroissement de ma puissance de persévérance, elle peut être considérée comme la manifestation consciente de ce qui, à un niveau métaphysique plus fondamental, est le désir ardent de persévérer et d'accroître mon pouvoir. Peut-être cela signifie-t-il, comme l'a soutenu Michael LeBuffe (From Bondage to Freedom: Spinoza on Human Excellence, Oxford/New York, Oxford University Press, 2010), qu'il faudrait désigner la conception spinozienne de la motivation par le terme « hédonisme » plutôt que par celui d' « égoïsme ». 
évalue le degré de correspondance d'une chose à une norme ou un modèle donnés. L'exemple le plus évident est celui de l'évaluation d'un artefact. Un bâtiment, par exemple, est considéré comme plus ou moins parfait selon qu'il est plus ou moins conforme à la conception originelle de l'architecte, qui sert de norme à laquelle le produit fini est comparé :

Qui a décidé de faire une certaine chose, et l'a faite jusqu'à son terme, ce n'est pas seulement lui-même qui dira que sa chose est parfaite, mais également quiconque aura correctement connu, ou cru connaître, ce qu'avait en tête l'Auteur de cette œuvre et quel était son but ${ }^{11}$.

Au contraire, si l'on ne sait pas ce que l'artisan a voulu créer, on n'aura aucun moyen d'évaluer le degré de perfection de l'artefact. Sans un modèle auquel comparer le produit, le terme « parfait» n'a donc aucun sens.

On peut appliquer cette analyse aux termes «bon» et «mauvais ». De la même manière, ces instruments d'évaluation doivent être compris dans le cadre de la relation d'une chose à une norme ou un modèle. Une chose est «bonne » si elle est un moyen efficace de parvenir à une fin. En particulier, dans la mesure où chaque individu s'efforce naturellement et nécessairement de maximiser sa puissance, une chose est «bonne » si elle favorise ce qui apparaît à l'individu comme son bien-être, et l'aide à se rapprocher de la condition idéale qui a été postulée ; et une chose est « mauvaise » si elle nuit à ce qui est perçu comme la puissance et le bien-être d'un individu. Il en résulte que «bon » et «mauvais », de même que «parfait» et «imparfait», sont des termes complètement relatifs, en l'occurrence, relatifs à la conception de l'intérêt d'un individu donné, et dans bien des cas ce qui est bon pour l'un peut ne pas l'être pour l'autre.

Il semblerait qu'ici, Spinoza aille au-delà de l'analyse du bien et du mal qu'il a menée auparavant. Dans la troisième partie, il écrit que l'on juge qu'une chose est bonne parce qu'on la désire (et on la désire parce qu'elle provoque une augmentation de la puissance d'agir) :

Quand nous nous efforçons à une chose, quand nous la voulons, ou aspirons à elle, ou la désirons, ce n'est jamais parce que nous jugeons qu'elle est bonne ; mais au contraire, si nous jugeons qu'une chose est bonne c'est précisément parce que nous nous y efforçons, nous la voulons, ou aspirons à elle, ou la désirons ${ }^{12}$.

Par conséquent, l'affirmation « $x$ est bon » doit signifier « je désire $x »$. De tels jugements sembleraient donc devoir être non seulement relativisés, mais également subjectivisés.

11. Éthique, op. cit., IV, préface.

12. Ibid., III, 9, scolie. 
Cependant, dans la préface de la quatrième partie, si les termes «bon » et «mauvais» sont relativisés, la question de savoir si un individu est conforme à un modèle idéal, ou si une chose, ou une action, mène à ce qui est considéré comme l'intérêt d'un individu, apparaît bien comme relevant d'un fait objectif. D'un autre côté, il semble que les normes ou modèles qui sont généralement utilisés pour déterminer le degré de perfection d'un individu, ou déterminer si une chose est «bonne » pour un individu, restent extrêmement subjectifs. La conception de ce qui constitue un arbre idéal, ou un être humain idéal, va différer d'une personne à une autre, en fonction de leurs expériences et donc des particuliers à partir desquels ils forment, par abstraction, leur notion générale, ou encore des caractéristiques auxquelles ils s'attachent au moment de la création de cette notion. Pour cette raison, il convient de faire référence à « ce qui est perçu comme » ou « ce qui apparaît comme » l'intérêt de quelqu'un; par exemple, une chose sera «bonne »si, étant donné ce que je crois être la vie idéale et les intérêts d'un individu, je crois que cette chose est bonne (c'est-à-dire qu'elle est un moyen d'atteindre cet idéal). Mais il n'y a aucune garantie que mes croyances soient vraies, ni que les autres les partagent. En effet, la formation de normes et de modèles dépend fortement des désirs particuliers d'un individu, de même que ses jugements quant à ce qui est bon et parfait ${ }^{13}$.

Si c'était là tout ce que Spinoza avait à dire sur le « bien » et le « mal », cela resterait, en effet, une analyse subjectiviste de ces termes moraux importants. Toutefois, il souligne que, même si le «bien » et le «mal» ne font pas référence à des caractéristiques réelles des choses, "nous devons cependant conserver ces vocables », tout en maintenant leur signification relative. Et ce, parce que, bien qu'ils restent relatifs à un contexte, Spinoza pense qu'ils peuvent aussi contenir une charge plus objective. Spinoza croit qu'il existe, de fait, un idéal spécifique qui peut servir de norme objective à partir de laquelle les choses peuvent être jugées comme vraiment «bonnes » pour l'être humain. Il y a un genre particulier de personne et de vie qui représente, objectivement, une perfection de la nature humaine :

Étant donné que nous désirons former une idée de l'homme à titre de modèle de la nature humaine que nous puissions avoir en vue, il nous sera utile de conserver ces mêmes vocables dans le sens que j'ai dit. Et donc, par bien, j'entendrai dans la suite ce que nous savons avec certitude être un moyen d'approcher toujours plus du modèle de la nature humaine que nous nous

13. Voir J. Bennett, A Study of Spinoza's Ethics, Indianapolis, Hackett Publishing, 1984, p. 292 : «La thèse selon laquelle nos jugements de valeur sont fondés sur des modèles n'entre pas en conflit avec la thèse précédente, selon laquelle ils sont guidés par nos sentiments et désirs. Bien plutôt, elles constituent deux aspects d'une unique conception unifiée, dont le point d'unification est l'idée que nos sentiments et désirs guident nos jugements de valeur en tant qu'ils nous guident, précisément, lorsque nous sélectionnons nos modèles (The thesis that our value judgments are based on models is not in conflict with the earlier thesis that they are guided by our feelings and desires. Rather, the two are aspects of a single unified account, the unifying factor being the view that our feelings and desires guide our value judgments by guiding our selection of models) ». 
proposons. Et, par mal, ce que nous savons avec certitude être un obstacle à ce que nous reproduisions ce même modèle. Ensuite, nous dirons les hommes plus parfaits ou plus imparfaits en tant qu'ils s'approchent plus ou moins de ce même modèle ${ }^{14}$.

Cela ne signifie pas que «bien » et «mal» ne soient pas des termes relatifs. Il est toujours vrai qu'aucune chose, considérée en elle-même, sans la comparer à une norme ou un modèle, n'est ni bonne ni mauvaise. Néanmoins, le subjectivisme introduit dans la troisième partie et prolongé dans la préface de la quatrième partie lorsque le bien et le mal sont réduits à des conceptions formées au hasard et destinées à servir de modèles à toutes sortes de choses (y compris les êtres humains), est à présent remplacé par un modèle plus objectif. «Bon» ne signifie plus simplement ce que l'on désire. Cela ne signifie pas non plus seulement «utile pour s'approcher de ce que quelqu'un croit être un spécimen perfectionné de son genre ». Désormais, cela signifie plutôt : utile à un homme pour se rapprocher de ce qui est, véritablement, un spécimen plus perfectionné de l'humanité ${ }^{15}$. Ce spécimen plus perfectionné de l'humanité consiste en un individu doté d'un maximum de puissance pour persévérer en tant qu'être humain, ou encore d'un maximum d'activité humaine. Une chose est «bonne » si elle contribue à accroître le conatus d'un individu, c'est-à-dire, si elle est l'objet du désir de quelqu'un qui ne croit pas seulement savoir ce qui est dans son intérêt, mais qui a la connaissance véritable de ce qui va effectivement augmenter sa puissance ou son effort.

Dans la quatrième partie, Spinoza définit donc le bien comme «ce que nous savons avec certitude nous être utile ${ }^{16}$ », et le mal comme « ce que nous savons avec certitude empêcher que nous possédions un bien ${ }^{17}{ }$. C'est aussi ce qu'il a à l'esprit lorsqu'il commence à parler de « la vraie connaissance du bien et du mal », par opposition à la simple «connaissance du bien et du mal», comme dans la proposition 14 de cette quatrième partie ${ }^{18}$. Cette dernière fait référence à la perception que j'ai d'une chose en tant qu'elle provoque un accroissement dans un aspect partiel de mes capacités. Certaines choses sont jugées «bonnes » parce qu'elles sont sources de joie et de plaisir. Par exemple, quelqu'un pourrait juger qu'un troisième verre de vin est «bon » parce que, pour une courte durée et au regard d'une partie seulement de sa puissance, ce verre le plonge dans un état de conscience agréable. Ce jugement n'est pourtant fondé que sur les passions, et par

14. Éthique, op. cit., IV, préface.

15. C'est pourquoi Jon Miller («Spinoza's Axiology », in Oxford Studies in Early Modern Philosophy, volume II, D. Garber S. Nadler [dir.], Oxford, Clarendon Press, 2005, p. 149-172) distingue rigoureusement parmi les valeurs relatives dont traite Spinoza, entre celle qui l'est au regard des circonstances et celle qui ne l'est pas au regard des circonstances.

16. Éthique, op. cit., IV, définition 1.

17. Ibid., IV, définition 2.

18. Ibid., IV, 8. 
conséquent s'appuie sur une connaissance inadéquate de la chose et de soi-même. En revanche, la «vraie connaissance du bien et du mal » est la perception rationnelle de ce qui m'est bénéfique, de manière plus complète et plus essentielle, portant ainsi véritablement mon individu entier à une condition plus puissante ; cette connaissance dérive d'idées adéquates et non de simples expériences hasardeuses, elle s'appuie sur la compréhension, et non sur la perception à courte vue de la manière dont une chose affecte positivement mon corps et mon esprit. Cette différence est résumée dans la démonstration de la proposition 35 de cette quatrième partie :

Ce que nous jugeons, sous la dictée de la raison, être bien ou mal, est nécessairement bien ou mal.

Dans son analyse du «bien» et du «mal», Spinoza ne semble pas s'intéresser aux notions d'action «correcte » (ou «droite ») ou «vicieuse » (ou « retorse »). Il évoque parfois la « façon correcte de vivre ${ }^{19} »$ et les actes «qu'on dit corrects ${ }^{20} »$, mais il n'explique pas en quoi consiste, exactement, la droiture de tels actes. Peut-être faut-il considérer que la conclusion qu'il tire à propos du «bon» doit s'appliquer aussi au «correct». Dès lors, une action sera « correcte » si elle conduit véritablement au bien-être de l'agent et provoque un accroissement de sa puissance ; une action est «vicieuse » si elle provoque une diminution de sa puissance. Il est sans doute possible qu'une même action puisse être correcte pour une personne et vicieuse pour une autre. Par exemple, plonger dans des eaux agitées pour sauver quelqu'un de la noyade peut être une action correcte pour un bon nageur, mais vicieuse pour quelqu'un qui ne sait pas nager. Cependant, il y a des choses qui sont correctes, ou bonnes, pour tous les êtres humains. Et ce, parce que nous partageons une certaine nature, en tant qu'êtres humains, et qu'il y a des actions qui contribuent naturellement et objectivement à la conservation et à l'effort de cette nature. De telles actions seront «vertueuses » dans un sens général, et seront accomplies par une personne vertueuse. Dans la mesure où Spinoza ne qualifie pas, en général, ces actions de «correctes », je parlerai, dans ce qui suit, des actions qui contribuent véritablement à la puissance d'un individu comme d'actions ayant une «valeur morale», ou encore comme d'actions « vertueuses » ou « bonnes ».

\section{La vertu}

La puissance ou l'effort d'un agent sont dirigés soit par l'expérience sensible et l'imagination (c'est-à-dire les «idées inadéquates »), soit par la connaissance («idées adéquates»). Lorsque le conatus ou désir d'un

19. Ibid., III, préface.

20. Ibid., III, déf. aff. 27, explication. 
individu est guidé par les sens et l'imagination, il recherche ce qu'il croit être bon pour lui, mais ce jugement est déficient; au contraire, lorsque son conatus est guidé par la connaissance, il accomplira de manière sûre et régulière ce qui augmente vraiment sa puissance d'agir.

Dans la quatrième partie, Spinoza définit la vertu, simplement, comme puissance :

Par vertu et puissance, j'entends la même chose, c'est-à-dire (par la Prop. 7 p. 3), la vertu, en tant qu'elle se rapporte à l'homme, est l'essence même ou nature de l'homme, en tant qu'il a le pouvoir de produire certains effets qui peuvent se comprendre par les seules lois de sa nature ${ }^{21}$.

Cette définition contient beaucoup de choses, et il est important de distinguer les différents éléments qui constituent la vie vertueuse selon Spinoza. Dans le scolie de la proposition 18, au détour d'un énoncé qui fait fortement écho à la doctrine stoïcienne de la vertu comme « action conforme à la nature », Spinoza offre une version plus condensée de la définition :

La vertu n'est rien d'autre qu' agir d'après les lois de sa propre nature ${ }^{22}$.

Mais la nature d'une chose n'est que son conatus, ou effort pour persévérer dans l'être. Par conséquent, les lois de la nature d'une chose prescrivent qu'elle conserve son être. Dès lors, Spinoza peut conclure :

Le fondement de la vertu est l'effort même pour conserver son être
propre $^{23}$.

Plus précisément, l'individu vertueux est celui qui suit les lois de sa propre nature et agit de façon à conserver son être propre. Pour le dire en un mot, la vertu n'est pas seulement l'exercice de la puissance, mais également l'effort de conservation qui réussit (ou bien, ce qui revient au même, l'exercice de la puissance informé par la connaissance) :

Plus chacun s'efforce de conserver son être et en a le pouvoir, plus il est doté de vertu ${ }^{24}$.

En revanche, le contraire de la vertu - que l'on supposerait être le vice, même si Spinoza n'utilise pas ce terme mais lui préfère celui d' «impuissance » - consiste à agir non selon les lois de sa propre nature, mais selon la nature des choses extérieures. L'individu dépourvu de vertu

21. Ibid., IV, definition 8.

22. Ibid., IV, 18, scolie.

23. Ibid.

24. Ibid., IV, 20, démonstration. 
souffre d'être conduit par les choses qui sont hors de lui, et qu'elles le déterminent à faire ce que réclame l'état commun des corps extérieurs, et non pas sa propre nature, considérée en $\operatorname{soi}^{25}$.

Jusqu'à présent, Spinoza a présenté une conception plutôt formelle de la vertu, qui n'a pas encore de réel contenu. L'affirmation "plus chacun s'efforce de rechercher ce qui lui est utile, c'est-à-dire de conserver son être, et en a le pouvoir, plus il est doté de vertu ${ }^{26} »$ n'indique pas de manière substantielle quel type de personnes s'efforce le mieux, et avec le plus de succès, vers l'autopréservation, ou comment agir de manière à suivre les lois de sa propre nature. Nous devons savoir ce qu'impliquent ces notions vagues de « suivre les lois de sa propre nature » et «s'efforcer de rechercher l'utile qui lui est propre », et comment une personne peut les mettre en œuvre dans sa vie.

C'est là que le rationalisme de Spinoza entre en scène, comme principe non plus seulement métaphysique ou épistémologique, mais moral. Spinoza identifie «vivre suivant les lois de sa propre nature» et «vivre sous la conduite de la raison ». Et ce, parce qu'un être humain vit selon les lois de sa propre nature lorsque ses actions ont leur cause adéquate dans sa nature seule, et non dans la manière dont les choses extérieures l'affectent; autrement dit, il vit suivant les lois de sa propre nature lorsqu'il est actif, et non passif. Et un être humain est actif - c'est-à-dire qu'il agit plutôt qu'il ne pâtit - lorsque ce qu'il fait suit de ses propres idées adéquates, de sa connaissance rationnelle des choses, et non des idées inadéquates ou des passions.

La conduite rationnelle ou raisonnable est contenue dans ce que Spinoza appelle «les prescriptions de la raison (dictamina rationis ${ }^{27}$ )». Ces prescriptions rationnelles sont fondées dans le conatus de l'individu et constituent une sorte d'expression propositionnelle éclairée de l'effort naturel. Elles réclament

que chacun s'aime lui-même, cherche ce qui lui est utile, ce qui lui est véritablement utile, et aspire à ce qui le mènera réellement à une plus grande perfection, et absolument parlant, que chacun s'efforce, autant qu'il est en lui, de persévérer dans son être ${ }^{28}$.

Plus important encore, la raison fournit des conseils sur la manière d'atteindre ces fins humaines communes. Elle le fait de manière universelle et objective, indépendamment des particularités des individus. Comme les impératifs catégoriques de la morale kantienne, les prescriptions de la raison transcendent les différences individuelles et formulent des exigences

25. Ibid., IV, 37, scolie 1.

26. Ibid., IV, 20.

27. Ibid., IV, 18, scolie.

28. Ibid. 
universelles pour la conduite humaine, dans la mesure où tous les êtres humains partagent la même nature fondamentale. Ceci est suggéré dans la proposition 72 de la quatrième partie : alors que Spinoza examine la question de savoir si l'individu qui vit sous la conduite de la raison pourrait agir de mauvaise foi, il affirme que «si la raison l'exigeait, elle l'exigerait pour tous les hommes ».

Une des premières choses que la raison réclame est que :

il faut aspirer à la vertu pour elle-même, et qu'il n'y a rien qui vaille mieux qu'elle ou ne soit plus utile ${ }^{29}$.

Mais, comme Spinoza l'a démontré dans les propositions métaphysiques de la première partie, nous sommes toujours nécessairement une partie de la Nature, et nous ne pouvons faire en sorte

de n'avoir pas besoin de quelque chose d'extérieur à nous pour conserver notre être, et de vivre sans avoir commerce avec les choses qui sont à l'extérieur de nous ${ }^{30}$.

C'est pourquoi la raison nous prescrit aussi de nous efforcer d'acquérir les nombreuses « choses qui sont hors de nous et nous sont utiles ${ }^{31} »$. Pour le dire autrement, la vertu, selon Spinoza, ne conduit pas à un retrait ascétique du monde, mais plutôt à une navigation plus intelligente et efficace dans le monde, et à un meilleur usage des choses. La personne vertueuse est capable de déterminer ce qui la conduit véritablement à son bien-être, et ce qui ne l'y conduit pas :

Agir absolument par vertu n'est rien d'autre qu'agir, vivre, et conserver son être (ce qui revient au même) sous la conduite de la raison, dont le fondement est la recherche de l'utile propre ${ }^{32}$.

Cette personne vertueuse discerne précisément son meilleur intérêt, elle désire et recherche activement ce qui va le mieux servir sa propre puissance de persévérer dans son être. En d'autres termes, elle sait ce qui est vraiment bon et s'efforce de l'acquérir.

Évidemment, l'éthique, telle qu'elle est ordinairement comprise, ne porte pas seulement sur l'autoconservation et le développement de soi. Elle doit également avoir quelque chose à dire sur la manière de traiter les autres êtres humains, même s'il se trouve qu'elle est motivée par la recherche de l'intérêt particulier. Spinoza a conscience de cela, et expose dans la quatrième partie de l'Éthique une brève discussion sur la manière dont un individu guidé par la raison agira nécessairement à l'égard des autres. S'il est 
certainement possible d'adopter une éthique égoïste et de ne tenir aucun compte du bien-être des autres dans la recherche illimitée de l'intérêt personnel, Spinoza montre de manière avisée qu'en réalité, l'égoïsme qui est au cœur de son système promeut ce que nous caractérisons, intuitivement, comme «éthique »: la bienveillance envers autrui et la prise en compte d'autrui. Mais si les actions sont altruistes, elles ne dérivent pas d'une motivation altruiste. Spinoza y insiste : il est dans l'intérêt de chacun de traiter les autres de telle sorte que leur propre puissance et effort soient accrus, et c'est cela qui motive l'action vertueuse. Autrement dit, la personne rationnelle (ou raisonnable) et vertueuse agira de façon à aider les autres à devenir rationnels (ou raisonnables) et vertueux uniquement parce qu'elle voit que les personnes qui sont comme elle, qui partagent sa nature, sont ce qui lui est le plus utile $\mathrm{e}^{33}$.

\section{L'action droite}

Nous pouvons à présent revenir à la question de l'importance de la motivation dans l'appréciation morale de l'action. En vertu de quoi une action droite est-elle «droite », pour Spinoza ? Il s'agit d'une action qui est «bonne », au sens de Spinoza - c'est-à-dire une action dont l'effectuation apporte un véritable bénéfice à l'agent et contribue à la préservation de son être ainsi qu'à l'augmentation de sa puissance. Ce qui revient à dire qu'une action a une valeur morale si et seulement si c'est une action dictée par la raison et en accord avec ses « prescriptions ». En d'autres mots, une action a une valeur morale si elle suit des idées adéquates et de la connaissance de l'agent, et qu'elle est, par conséquent, vertueuse. Et ce, parce qu'une telle action consiste en l'effort efficace de se conserver et d'accroître sa puissance.

Il semblerait donc que la motivation d'un agent ne devrait pas avoir de relation avec le statut moral de l'action, c'est-à-dire avec le fait qu'elle soit bonne ou mauvaise. Aider ceux qui sont dans le besoin, contribuer à l'épanouissement et au bien-être des autres devrait être dans l'intérêt de chacun - cela devrait aider la puissance et l'effort de chacun en permettant de créer les conditions dans lesquelles chacun est entouré d'autres individus qui partagent sa nature, et donc qui sont ce qui lui est le plus utile indépendamment de ce qui pousse l'agent à s'engager dans une action aussi bienveillante. Comme le souligne Spinoza, une même action peut naître de passions irrationnelles ou être l'effet d'une conception rationnelle :

À toutes les actions auxquelles nous détermine un affect qui est une passion, nous pouvons être déterminés sans lui par la raison ${ }^{34}$.

33. Voir Éthique, IV, propositions 31 à 37.

34. Ibid., IV, 59. 
L'action bienveillante envers autrui peut naître aussi bien de la pitié que de la vertu, autrement dit d'une motivation émotionnelle aussi bien que d'un désir guidé par la connaissance de ce qui nous est véritablement utile ; et il semblerait que cela ne fasse aucune différence au regard de la qualité morale de l'action elle-même ${ }^{35}$. Elle reste bonne, droite, dans la mesure où elle est dans l'intérêt de celui qui l'accomplit.

Dans cette perspective, il est important de clarifier le lien entre vertu et action. Au final, il se pourrait que les actions vertueuses ne puissent être accomplies que par les individus vertueux, et que les actions moralement valables ne puissent suivre que de la raison et des idées adéquates ; peut-être les passions et les idées inadéquates conduisent-elles toujours à se méprendre sur l'utile véritable. Et il se pourrait que les idées adéquates soient un guide infaillible vers ce qui est véritablement utile et que les personnes vertueuses n'accomplissent que des actions droites. Mais tout cela reviendrait à ne prendre en considération que les éléments causaux. Nous pouvons même dire qu'une action droite n'est rien d'autre que l'action que la personne vertueuse, guidée par la raison sur la base d'idées adéquates, désire (c'est-à-dire est déterminée à) faire. Cela n'établirait toujours pas que la valeur morale de l'action soit fonction de sa motivation, ni qu'une action ne soit vertueuse que lorsqu'elle est conforme à ce qu'une personne vertueuse ferait. Ce qui fait qu'une action vertueuse est bonne, dans cette lecture de la doctrine de Spinoza, consiste précisément en ce que la personne sait que l'action mène à un accroissement de sa puissance.

\section{Motivation et capacité à agir}

Cependant, tout n'est pas ce qu'il semble être. L'interprétation que je viens de décrire, si tentante soit-elle, réduit considérablement ce que Spinoza dit de la capacité d'agir, et occulte notamment la manière dont il pense la relation entre action, vertu, idées adéquates et puissance. En fait, il apparaît qu'au moins dans certains cas très importants, la motivation de l'agent c'est-à-dire les croyances et les désirs qui sont à l'origine de l'action - est cruciale pour déterminer la qualité morale de l'action, pour juger si, oui ou non, l'action est «bonne » au sens de Spinoza, c'est-à-dire au sens où elle conduit à un accroissement de la puissance de l'agent ${ }^{36}$.

Pour le comprendre, revenons au cas de la bienveillance qui a été brièvement évoqué tout à l'heure. En premier lieu, rappelons ce que Spinoza écrit dans la proposition 59 de la partie IV :

35. À cet égard, on peut comparer ce qu'il écrit sur la bienveillance à la fin de la troisième partie (Ibid., III, déf. aff. 35) et la conception de la bienveillance développée dans les propositions 31 à 36 de la quatrième partie.

36. Mon analyse est ici redevable à la discussion avec Matt Kisner, et je lui suis reconnaissant de sa perspicacité sur les conceptions de Spinoza. 
À toutes les actions auxquelles nous détermine un affect qui est une passion, nous pouvons être déterminés sans lui par la raison ${ }^{37}$.

Une même action peut naître soit d'une passion, soit de la raison et de la connaissance. Tout dépend si le désir d'accomplir l'action se forme à partir d'idées adéquates ou inadéquates, d'une vraie compréhension ou d'une croyance incomplète concernant la contribution de cette action à la fortification du conatus. Ceci est vrai des actions que l'on pourrait accomplir pour le bénéfice d'autres individus. Spinoza écrit explicitement que l'on peut agir avec bienveillance envers les autres soit par pitié ou compassion, soit en étant motivé par des idées adéquates, parce que l'on saisit en quoi traiter autrui de manière juste et charitable contribue à l'épanouissement de soi-même. J'avais proposé tout à l'heure, comme interprétation provisoire de cette proposition 59 de la quatrième partie, qu'elle suggérait que la motivation n'était pas à prendre en compte dans l'évaluation morale de l'action ; en fait, pour Spinoza, cette proposition implique tout le contraire.

Dans le scolie de la proposition 22 de la troisième partie, Spinoza définit la pitié comme «une Tristesse née du malheur d'autrui ». Il explique plus loin qu'elle est fondée sur ce qu'il appelle l'« imitation des affects », qui est une réaction d'empathie :

De ce que nous imaginons une chose semblable à nous, que nous n'avons poursuivie d'aucun affect, affectée d'un certain affect, nous sommes par là-même affectés d'un affect semblable ${ }^{38}$.

Si la «chose » est une autre personne (et donc très semblable à nous), et si cette autre personne est affectée de tristesse - c'est-à-dire, d'une diminution de sa puissance d'agir -, alors nous serons également affectés de tristesse et éprouverons une diminution de puissance correspondante : Pitié ${ }^{39}$.

Cette imitation des affects, quand elle se rapporte à la Tristesse, s'appelle

La pitié ainsi comprise est un affect passif (ou passion) parce qu'elle consiste en une diminution de ma puissance causée par une chose extérieure (ou plutôt la perception que j'en ai).

Cette pitié peut dès lors donner naissance au désir d'aider l'autre personne :

Cette volonté ou appétit de faire du bien, qui naît de ce que nous fait pitié la chose à laquelle nous voulons faire du bien, s'appelle Bienveillance ${ }^{40}$.

37. Éthique, op. cit., IV, 59.

38. Ibid., III, 27.

39. Ibid., III, 27, scolie.

40. Ibid., III, 27, corollaire 3, scolie. 
Un acte de bienveillance qui advient sous l'impulsion de la pitié relève toujours d'une diminution de la puissance d'agir. Il n'est donc pas bon, mais mauvais. Agir par pitié, même si cela bénéficie à autrui, n'aide pas mon effort mais plutôt, s'y oppose. C'est une action qui est fondée sur des idées inadéquates et motivée par elles.

Toutefois, comme nous l'avons vu, les actes de bienveillance peuvent également naître non pas des passions et des idées inadéquates, mais de la connaissance et des idées adéquates. Dans ce cas, une personne rationnelle et vertueuse vient en aide à une autre non parce qu'elle ressent de la tristesse mais parce qu'elle comprend clairement que c'est dans son intérêt de le faire. Elle sait qu'une personne épanouie, rationnelle et vertueuse a une nature qui est plus semblable à la sienne et donc lui est plus utile; et cette connaissance la pousse à contribuer à la vertu et la rationalité de cette autre personne. Dans la proposition 50 de la quatrième partie, Spinoza remarque qu'un individu qui vit «sous la conduite de la raison» n'aura pas besoin de pitié, et sait que

le bien qui suit d'elle [la pitié], à savoir que nous nous efforçons de délivrer de son malheur l'homme qui nous fait pitié (par le Coroll. 3 Prop. 27 p. 3), c'est sous la seule dictée de la raison que nous désirons le faire (par la Prop. 37 de cette p.), et ce n'est que sous la seule dictée de la raison que nous pouvons faire quelque chose que nous savons avec certitude être bon ${ }^{41}$.

L'acte bienveillant de la personne rationnelle (ou raisonnable), contrairement à l'acte bienveillant accompli par pitié, ne consiste pas en une tristesse mais en une joie. Il indique non une diminution, mais un accroissement de la puissance d'agir ; il est donc une action vertueuse.

Cela s'explique, en particulier, parce qu'accomplir une action qui suit d'idées adéquates, comme le fait l'individu rationnel (ou raisonnable), n'est rien d'autre qu'éprouver un accroissement de sa puissance ${ }^{42}$. Spinoza note qu'à proprement parler, l'esprit n'agit que lorsqu'il est la cause adéquate, et non partielle, d'un effet; et cela se produit lorsque le désir est mû par des idées adéquates (alors qu'il pâtit, ou est passif, lorsque le désir est mû par ses idées inadéquates, c'est-à-dire par la manière dont les choses extérieures affectent son $\operatorname{corps}^{43}$ ). Agir, c'est exprimer sa puissance. Exprimer sa puissance, c'est en cela que consiste vertu. Par conséquent, le plus vertueux est celui qui agit selon des idées adéquates. En ce sens, Spinoza définit, d'un point de vue pratique, l'action vertueuse comme une action motivée d'une certaine manière (en l'occurrence, par des idées adéquates).

41. Ibid., IV, 50, démonstration.

42. Voir M. J. Kisner, Spinoza on Human Freedom: Reason, Autonomy and the Good Life, Cambridge, Cambridge University Press., 2011, p. 142-145.

43. Éthique, op. cit., III, 1. 
Il s'ensuit donc que se montrer bienveillant envers les autres en étant motivé par la raison et les idées adéquates est une action vertueuse; en revanche, se montrer bienveillant envers les autres en étant motivé par la pitié n'est pas une action vertueuse, mais mauvaise. Ce que dit Spinoza dans l' «autre démonstration » de la proposition 59 de la quatrième partie sur les actions en général et les affects de haine s'applique en particulier à l'action bienveillante et l'affect de pitié (qui, comme la haine, est un affect «mauvais » dans la mesure où elle affaiblit la puissance) :

Une action quelconque est dite mauvaise en tant qu'elle naît de ce que nous sommes affectés de Haine ou de quelque affect mauvais. Or aucune action, considérée en soi seule, n'est bonne ou mauvaise (comme nous l'avons montré dans la Préface à cette Partie) : mais une seule et même action est tantôt bonne, tantôt mauvaise ; donc à cette même action qui présentement est mauvaise, autrement dit qui naît d'un affect mauvais, nous pouvons être conduits par la raison (par la Prop. 19 de cette p.).

Remarquons que Spinoza identifie ici « une action qui est mauvaise » à « une action qui naît d'un affect mauvais ». Puisque c'est l'affect qui motive l'action, pour Spinoza, cela équivaut à dire que la qualité morale d'une action est fonction de sa motivation. Il n'est pas seulement question, ici, de porter un jugement sur l'agent - à savoir, qu'un agent n'agit vertueusement que s'il agit suivant la bonne motivation - ni même de porter un jugement sur l'accomplissement vertueux d'une action, mais bien de porter un jugement sur l'action elle-même.

La même analyse s'applique à d'autres types d'action. Prenons, par exemple, la retraite d'un individu au cours d'une bataille. En elle-même, la retraite au cours d'une bataille n'est ni bonne ni mauvaise ; elle n'est qu'un certain type de mouvement physique. Toutefois, si la motivation de la retraite est la peur, que Spinoza considère comme une espèce de tristesse ${ }^{44}$, alors l'action (la retraite) sera mauvaise, puisqu'elle implique un affaiblissement de la puissance de l'individu. En revanche, la motivation de la retraite face au danger, chez une personne qui vit sous la conduite de la raison, est la «fermeté (animositas) », définie comme «le Désir par lequel chacun s'efforce de persévérer dans son être sous la seule dictée de la raison $^{45}$ ", que l'on peut également traduire par «force d'âme» ou «présence d'esprit ». Grâce à sa fermeté, un individu apprécie correctement le danger, en le rapportant à sa propre capacité à le surmonter, et donc sait « fuir à temps » lorsque cela est nécessaire à la conservation de son être ${ }^{46}$. La retraite face au danger mue par la peur est mauvaise, mais la retraite face au danger mue par la fermeté est vertueuse : il s'agit du même mouvement, mais de motivations différentes, et donc de différents statuts moraux.

44. Ibid., III, 18, scolie 2.

45. Ibid., III, 59, scolie.

46. Ibid., IV, 69. 
Il y a un passage éloquent de la correspondance de Spinoza, dans une lettre adressée à Willem van Blyenbergh en 1665, dans laquelle Spinoza considère la question de savoir si Dieu, selon sa conception métaphysique (qui trouble profondément van Blyenbergh), «agrée également le meurtre et l'aumône ${ }^{47}$ ». Voici ce qu'il écrit :

S'agit-il de savoir si l'une et l'autre ouvre, en tant qu'elles sont quelque chose de réel dont Dieu est cause, sont également parfaites? Je réponds que, si nous considérons uniquement les œuvres de cette manière, il peut se faire qu'il y ait dans l'une et l'autre une perfection égale. Demandez-vous si le voleur et le juste sont également parfaits, ont la même béatitude ? Je réponds : non. Par juste, en effet, j'entends celui qui désire constamment que chacun ait ce qui lui revient et, dans mon Éthique (non encore publiée), je démontre que ce désir, chez les hommes pieux, tire nécessairement son origine de la connaissance claire qu'ils ont d'eux-mêmes et de Dieu. Le voleur n'ayant pas un désir de cette sorte, il est donc nécessairement dépourvu de la connaissance de Dieu et de lui-même, c'est-à-dire de ce qui d'abord nous fait hommes ${ }^{48}$.

Selon l'éthique ordinaire, tuer ou voler quelqu'un est une mauvaise chose ; faire l'aumône et traiter les autres avec justice est une bonne chose. Ce que Spinoza répond à Blyenbergh est que le caractère bon et mauvais de ces actions ne réside pas dans les actions «considérées seules», indépendamment de leurs motivations - puisque dans cette perspective elles sont «également parfaites », c'est-à-dire contiennent la même quantité de réalité ; mais ce caractère bon ou mauvais réside dans le fait que l'une dérive d'idées adéquates et l'autre de l'ignorance, et donc, que l'une consiste en un accroissement de la puissance de l'agent et l'autre, en une diminution de cette puissance.

Nous pouvons donc conclure que, pour Spinoza, il y a beaucoup de cas - et peut-être est-ce vrai pour toutes les actions sujettes à une évaluation morale - où la qualité morale d'une action est indéterminée tant que la motivation de l'agent n'est pas prise en compte ${ }^{49}$. Pour ne prendre qu'un

47. Lettre 23 (Spinoza, Lettres, trad. C. Appuhn, in CEuvres IV, Paris, Garnier Frères, 1966).

48. Ibid.

49. Les conclusions méta-éthiques que j'ai formulées ici peuvent également être tirées de son ouvrage antérieur, le Court Traité (Spinoza, Court traité, trad. J. Ganault, in Euvres I. Premiers écrits, Paris, PUF, 2009). Après avoir remarqué que «nous accomplissons nos actions de deux manières, à savoir avec ou sans passions » (II, VI, 1), Spinoza conclut que «si nous réalisons sans passion les choses que nous avons à faire, aucun mal ne peut en provenir. Et puisqu'entre bien et mal il n'est pas d'intermédiaire, nous voyons donc que s'il est mal d'agir avec passion, il doit être bien d'agir sans elles» (II, VI, 2). Par exemple, bien que le remords et le repentir (qui sont des passions) puissent mener une personne vers «le droit chemin » et donc sembler bons, Spinoza écrit que «si nous les examinons correctement, nous verrons que non seulement ils ne sont pas bons, mais au contraire qu'ils sont néfastes et par suite mauvais. Car il est évident que nous parvenons toujours mieux à la vérité par la raison et l'amour, que par le remords et le repentir. Ils sont néfastes et donc mauvais, parce qu'ils sont une espèce de tristesse, de laquelle nous avons déjà démontré qu'elle était néfaste et que, comme mauvaise, nous devions pour cela tendre à l'éloigner de nous; nous devons de même, par suite, les craindre et les fuir » (II, X, 2). 
exemple remarquable, les actions bienveillantes à l'égard d'autrui n'augmentent pas véritablement la puissance, donc ne sont pas «bonnes », à moins de naître d'une certaine motivation, à savoir la condition épistémique et affective caractéristique de l'individu vertueux et rationnel (ou raisonnable). Comme le dit Spinoza, dans un énoncé qui révèle à quel point il importe de tenir compte de la motivation dans l'évaluation :

Le Désir de faire du bien qu'engendre en nous le fait que nous vivions sous la conduite de la raison, je l'appelle Moralité ${ }^{50}$.

Texte traduit de l'anglais par Sophie Laveran

50. Éthique, op. cit., IV, 37. 


\section{Bibliographie}

\section{Cuvres de Spinoza}

Spinoza Opera, 5 vol., Heidelberg, Carl Winters Universitaetsbuchandlung, 1925.

Court traité, trad. J. Ganault, in Euvres I. Premiers écrits, Paris, PUF, 2009. Lettres, trad. C. Appuhn, in Euvres IV, Paris, Garnier Frères, 1966.

Éthique, trad. B. Pautrat, Paris, Seuil, 1988.

\section{Commentaires}

Bennett, J., A Study of Spinoza's Ethics, Indianapolis, Hackett Publishing, 1984.

Curley, E., «Spinoza's Moral Philosophy », in Spinoza: A Collection of Critical Essays, Grene, M. (dir.), Notre Dame, University of Notre Dame Press, 1973, p. 354-376.

Curley, E., Behind the Geometric Method, Princeton, Princeton University Press, 1988.

Della Rocca, M., «Egoism and the Imitation of the Affects in Spinoza », in Spinoza on Reason and the Free Man, Yovel, Y. et Segal, G. (dir.), New York, Little Room Press, 2004, p. 123-148.

Garrett, D., «Spinoza's Ethical Theory », in The Cambridge Companion to Spinoza, Garrett, D. (dir.), Cambridge, Cambridge University Press, 1996, p. 267-314.

Kisner, M. J., Spinoza on Human Freedom: Reason, Autonomy and the Good Life, Cambridge, Cambridge University Press, 2011.

LeBuffe, M., From Bondage to Freedom: Spinoza on Human Excellence, Oxford/New York, Oxford University Press, 2010.

Miller, J., "Spinoza's Axiology », in Oxford Studies in Early Modern Philosophy, volume II, Garber, D. et Nadler, S. (dir.), Oxford, Clarendon Press, 2005, p. 149-172.

Sverdlik, S., Motive and Rightness, Oxford, Oxford University Press, 2001. 